\title{
Self-propelled swimming of a flexible filament driven by coupled plunging and pitching motions *
}

\author{
Bing-lin Li ${ }^{1,3}$, Yi-wei Wang ${ }^{2,3}$, Bo Yin ${ }^{2,3}$, Xiang Zhang ${ }^{1,3}$, Xing Zhang ${ }^{1,3}$ \\ 1. The State Key Laboratory of Nonlinear Mechanics, Institute of Mechanics, Chinese Academy of Sciences, \\ Beijing 100190, China \\ 2. Key Laboratory for Mechanics in Fluid Solid Coupling Systems, Institute of Mechanics, Chinese Academy of \\ Sciences, Beijing 100190, China \\ 3. School of Engineering Science, University of Chinese Academy of Sciences, Beijing 100049, China
}

(Received December 11, 2019, Revised June 9, 2020, Accepted June 21, 2020, Published online March 5, 2021) (C) China Ship Scientific Research Center 2021

\begin{abstract}
This paper numerically investigates the self-propelled swimming of a flexible filament driven by coupled pitching and plunging motions at the leading edge. The influences of bending rigidity and some actuation parameters (including the phase offset between pitching and plunging, and the amplitudes of pitching and plunging motions) on the swimming performance are explored. It is found that with increasing rigidity, the swimming style gradually transits from the undulatory mode to the oscillatory mode. The plunging-pitching actuation is found to be superior to the plunging-only actuation, in the sense that it prevents the decrease of speed at high rigidity and achieves a higher efficiency across a wide range of rigidity. The comparison of the body kinematics with those of animal swimmers, and the classification of the wake structures are discussed. The results of this study provide some novel insights for the bio-inspired design of autonomous underwater vehicles.
\end{abstract}

Key words: Self-propelled swimming, flexible filament, fluid-structure interaction (FSI), vortex structures, immersed boundary method

\section{Introduction}

The role of passive flexibility in bio-locomotion has received an increasing attention recently from the communities of biologists and engineers. For biological propulsors, passive flexibility makes a great contribution in producing the deformation during the swimming or the flying. The proper "mixing" of active control and passive flexibility is believed to be the key in optimizing the locomotion performance. For the design of bio-inspired swimmers, the utilization of passive flexibility can significantly reduce the complexity of active control in the actuation system.

To gain a physical insight into the hydrodynamic effect of passive flexibility, some highly simplified bio-locomotion models were developed. A

* Project supported by the National Natural Science Foundation of China (Grant Nos. 11772338, 11372331).

Biography: Bing-lin Li (1993-), Male, Master,

E-mail: binglinli106@126.com

Corresponding author: Xing Zhang,

E-mail: zhangx@1nm.imech.ac.cn self-propelled flexible filament (or panel) actuated locally at the leading edge is a model that has been extensively studied. In this model, the kinematics of the body (excluding the leading edge) is determined by the fluid-structure interaction (FSI), and is not prescribed. Here we might stress that in this model the motion in the swimming direction is unrestricted ${ }^{[1-2]}$. The free-swimming (or self-propelled) condition in this model distinguishes it from the model in which a "tethered" flexible body is placed in an incoming flow and forced to oscillate. The model of the latter type is very dissimilar to a biological swimmer and thus the studies based on such model will not be covered in this literature review. We notice that more complex models with the full geometry of angulliform swimmers have also been studied ${ }^{[3-4]}$. The studies related to this kind of models will not be discussed here either.

Among the studies based on this simplified model, the most intensively investigated actuation is the sinusoidal plunging motion. The effects of some key parameters, including the plunging amplitude, the bending rigidity, the mass ratio, the thickness, the shape and the Reynolds number, on the swimming performance have been systematically investigated for 
a flexible 2-D filament ${ }^{[5-8]}$ or a flexible 3-D panel ${ }^{[9-10]}$. All results indicate that a moderate flexibility is beneficial for enhancing the swimming performance (in terms of the cruising speed and efficiency). The relation between the structural resonance and the performance optimization, and the effect of flexibility on the wake symmetry properties were addressed in some papers ${ }^{[6-7,9-11]}$. It should be noted that the above studies focused on the deformation of the first mode (or the oscillatory swimming style as adopted by tuna and dolphin).

Another type of actuation, namely, the coupled motions of (sinusoidal) plunging and pitching, was mainly used for reproducing the deformation of a higher mode (or the undulatory swimming style as adopted by eel, stingray and knifefish). In a series of experiments conducted by Lauder and coworkers at Harvard University[12-17], the influences of some key parameters (such as the length, the shape and the stiffness) on the deformation pattern and the swimming performance were studied. The swimming performances of the two aforementioned actuations (i.e., the plunging-only one and the plunging-pitching one) were also compared in Lauder et al. ${ }^{[13-14]}$. It was found that in the case of the plunging-only actuation, the cruising speed could reach the peak at one rigidity value and then decreases. However, in the case of the plunging-pitching actuation, a broad plateau in the cruising speed could be reached when the rigidity exceeds a certain value. In the work by Ramananarivo et al. ${ }^{[18]}$, it was found that the energy dissipation due to the interaction with the surrounding fluid was essential for the development of a traveling wave in the body kinematics (one typical feature of the anguilliform swimming). In the studies by Dai et al. ${ }^{[19-20]}$, the carangiform swimming style was successfully reproduced by tuning the non-uniformly distributed rigidity and mass ratio. The influence of the phase offset between the plunging and pitching motions on the swimming performance was addressed by Kim et al. ${ }^{[21]}$ and Piñeirua et al. ${ }^{[22]}$.

Despite of the insights gained by the aforementioned studies, the knowledge of the swimming performance of the plunging-pitching actuation is far from complete. First, the performances of the plunging-only and plunging-pitching actuations have not been systematically compared across a wide range of the bending rigidity. Second, little is known about how to properly combine the plunging and pitching motions to achieve the optimal performance. The inconsistency in some previous studies regarding the optimal phase offset between the plunging and pitching motions needs to be clarified in particular. Third, a quantitative comparison between the kinematics of the elastic swimmer and those of animal swimmers is still lacking. In this work, we use high- fidelity numerical simulation and broad parametric sweeps to tackle this problem. Based on the simulation results, the ideal parameters for optimizing the swimming performance are suggested.

\section{Computational model and governing equations}

We consider the self-propelled swimming of a flexible filament in a 2-D fluid flow. The swimming of the filament is actuated by the coupled plunging and pitching motions prescribed at the leading edge (see Fig. 1 for a schematic diagram of the computational model). The driving motions can be described in a mathematical form as:

$$
\begin{aligned}
& y_{\mathrm{LE}}(t)=A \cos (2 \pi f t) \\
& \theta_{\mathrm{LE}}(t)=A_{\theta} \cos (2 \pi f t-\Delta \phi)
\end{aligned}
$$

where $y_{\mathrm{LE}}$ and $\theta_{\mathrm{LE}}$ are the vertical and angular displacements of the leading edge, respectively. $A$ and $A_{\theta}$ are the amplitudes of the plunging and pitching motions, respectively. $f$ is the flapping frequency and $\Delta \phi$ is the phase offset between the pitching and the plunging motions.

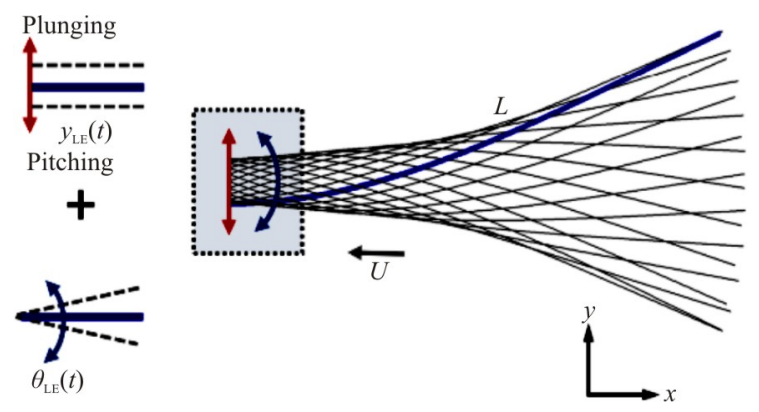

Fig. 1 (Color online) A schematic diagram of the computational model

The flow around the filament is assumed to be laminar and incompressible. The Naiver-Stokes equations which govern the fluid motion can be written in a dimensionless form as:

$$
\frac{\partial \boldsymbol{u}}{\partial t}+\nabla \cdot(\boldsymbol{u u})=-\nabla p+\frac{1}{R e_{f}} \nabla^{2} \boldsymbol{u}+\boldsymbol{f}
$$

$\nabla \cdot \boldsymbol{u}=0$

where $\boldsymbol{u}$ is the fluid velocity, $p$ is the pressure, $\boldsymbol{f}$ is the dimensionless Eulerian force which represents the effect of an immersed body on the fluid flow. The flapping Reynolds number in Eq. (3) is 
defined as $R e_{f}=U_{\text {ref }} L / v$, where $U_{\text {ref }}, L$ and $v$ are the reference velocity, the body length and the kinematic viscosity of the fluid, respectively. Here the reference velocity is defined as $U_{\text {ref }}=L f$.

The governing equations for the motion of the filament can be written in a dimensionless form as:

$$
\begin{aligned}
& \beta \frac{\partial^{2} \boldsymbol{X}}{\partial t^{2}}-\frac{\partial}{\partial s}\left[\xi(s) \frac{\partial \boldsymbol{X}}{\partial s}\right]+\frac{\partial^{2}}{\partial^{2} s}\left(\gamma \frac{\partial^{2} \boldsymbol{X}}{\partial s^{2}}\right)=-\boldsymbol{F} \\
& \frac{\partial \boldsymbol{X}}{\partial s} \cdot \frac{\partial \boldsymbol{X}}{\partial s}=1
\end{aligned}
$$

where $\boldsymbol{X}$ is the position vector for the Lagrangian marker point on the filament, $s$ is the Lagrangian coordinate along the arc length, $\boldsymbol{F}$ is the dimensionless Lagrangian forcing which represents the interaction between the filament and the fluid.

The dimensionless quantities $\beta, \xi$ and $\gamma$ in

Eq. (5) are the mass ratio, the dimensionless tension coefficient and the dimensionless bending rigidity, respectively. The definitions of these dimensionless quantities are:

$\beta=\frac{\rho_{s} \delta}{\rho_{f} L}, \quad \xi=\frac{T}{\rho_{f}\left(U_{r e f}\right)^{2} L}, \quad \gamma=\frac{B}{\rho_{f}\left(U_{\text {ref }}\right)^{2} L^{3}}$

where $\rho_{f}$ and $\rho_{s}$ are the densities of the fluid and the filament, respectively. $T$ and $B$ are the (dimensional) tension coefficient and the (dimensional) bending rigidity, respectively. It should be noted that the equations which govern the motion of the filament are nonlinear due to the inhomogeneity in the dimensionless tension coefficient $\xi$. This tension coefficient is also used to enforce the inextensibility condition (Eq. (6)). The governing equation for $\xi$ can be derived by rearranging Eqs. (5), (6). The values of $\xi$ are then obtained by numerically solving a boundary value problem ${ }^{[23]}$.

\section{Numerical methods and settings}

The immersed boundary method based on the discrete stream function formulation is used to solve the Navier-Stokes equations (Eqs. (3), (4)) ${ }^{[24-25]}$. The structure equations (Eqs. (5), (6)) are solved by using a finite difference method for the spatial discretization. A three-time-level scheme is used for the temporal advancement, in which the tension term is treated explicitly and the bending term is treated implicitly. The FSI simulation is performed by loosely coupling the two different solvers, i.e., alternately advancing the fluid equations and the structure equations. This
FSI solver has been thoroughly validated and was successfully used in the previous studies on the selfpropelled swimming of an elastic swimmer ${ }^{[6,19-20,26]}$.

The computational domain in this study is a rectangular box with the size of $[-23 L, 23 L] \times$ $[-6 L, 6 L]$. This computational domain is divided into seven subdomains and a multi-block Cartesian mesh is generated for the simulation (see Fig. 2). The highest mesh resolution of $L / 200$ is used in the innermost subdomain of $[-14 L, 14 L] \times[-0.6 L, 0.6 L]$, surrounding the filament. Lower resolutions are used in the outer subdomains. For the outermost subdomain, the mesh resolution is reduced to $16 L / 75$. The time step is chosen such that the maximum Courant-FriedrichsLewy (CFL) number never exceeds 0.5 .

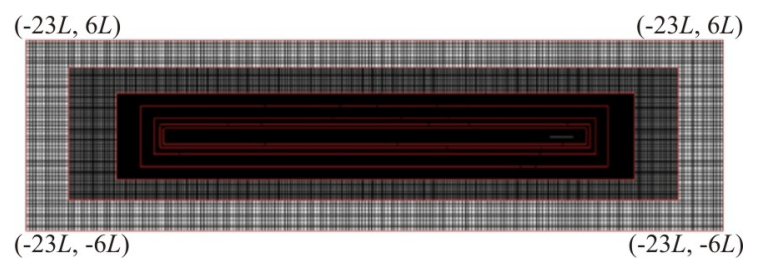

Fig. 2 (Color online) The computational domain and the multiblock Cartesian mesh used in the numerical simulation. Seven subdomains with different mesh resolutions are shown. The gray line in the innermost subdomain represents the initial position of the filament

Since the unknowns to be solved here are the stream functions (rather than the velocities), the conservation of the velocity flux at the interfaces between the coarse and fine meshes can be automatically satisfied. The decrease of the accuracy and the distortion in the vorticity field are found locally at such interfaces, because of the irregular dual cells near the hanging nodes for performing the discrete curl $(\nabla \times)$ operation ${ }^{[24]}$ (see Fig. 1 of Ref. [24]). However, the effect of such interfaces on the overall accuracy is very limited. First, the number of the hanging nodes is very small. Second, such interfaces are located at the places far away from the swimmer.

The no-slip boundary condition is enforced on the four sidewalls of the computational domain. The non-slip boundary on the filament is imposed by using the direct-forcing immersed boundary technique ${ }^{[24]}$. For the structure equations, the free-end boundary condition is imposed at the trailing edge. At the leading edge, both the prescribed plunging and pitching motions and the horizontally unrestricted condition are enforced simultaneously[ ${ }^{[6]}$. The initial velocities for the fluid and the filament are set to be zero. The initial configuration of the filament is a flat plate with the leading edge placed at $x=12 L$ and 
$y=A$.

A mesh sensitivity study is conducted by simulating a testing case. The control parameters for this case are: $\gamma=0.05, \beta=0.01, A / L=0.05$, $A_{\theta}=30^{\circ}, R e_{f}=300$. Three meshes of different resolutions are generated for the study (see Table 1 for the detailed information).

Table 1 The information for the meshes used in the mesh sensitivity test

\begin{tabular}{cccc}
\hline Mesh & $\begin{array}{c}\text { Mesh resolution } \\
(L)\end{array}$ & $\begin{array}{c}\text { Number of grid } \\
\text { points }\end{array}$ & $\begin{array}{c}\text { Number of } \\
\text { blocks }\end{array}$ \\
\hline C & $1 / 100-4 / 25$ & $0.43 \times 10^{6}$ & 5 \\
I & $1 / 300-16 / 75$ & $3.24 \times 10^{6}$ & 7 \\
F & $1 / 400-4 / 25$ & $6.62 \times 10^{6}$ & 7 \\
\hline
\end{tabular}

Note: C-Coarse mesh, I-Intermediate mesh, F-Finest mesh.

The time histories of the horizontal velocity at the leading edge and the instantaneous lateral and horizontal forces, which are obtained by using the three meshes, are shown in Figs. 3, 4, respectively. From these figures, the mesh convergence can be clearly seen. The difference between the results obtained with the intermediate and the finest meshes is rather small. As a compromise between accuracy and computational cost, the intermediate mesh is used in the present study.

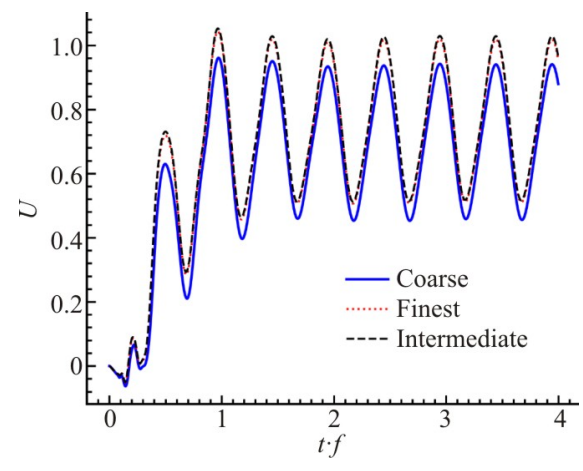

Fig. 3 (Color online) Time histories of the horizontal velocity of the leading-edge that are obtained by using three meshes of different resolutions

From Fig. 4, a high-frequency oscillation of the lateral force is seen from the results obtained with the coarse mesh. This oscillation is a numerical artifact which does not reflect the real physics. Such spurious oscillation can be suppressed by either reducing the grid width or increasing the width of the interpolation kernel (i.e., the discrete $\delta$-function) used in the immersed boundary method ${ }^{[27]}$. It should be emphasized here that for the mesh resolution used in the simulation, the spurious oscillation is rather small and has no important effect on the accuracy of the force prediction.
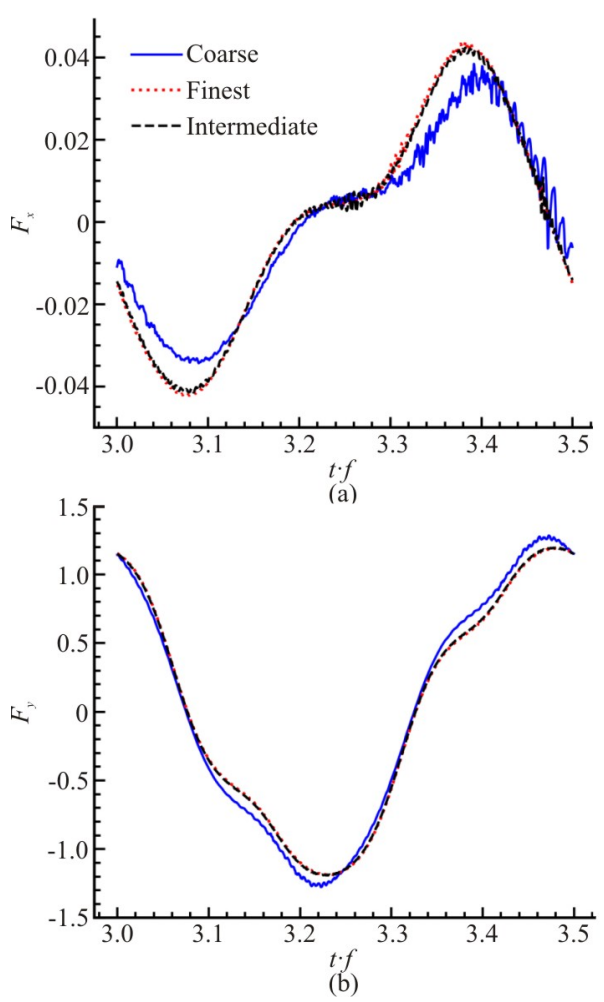

Fig. 4 (Color online) Time histories of the instantaneous lateral force (a) and instantaneous horizontal force (b) that are obtained by using three meshes of different resolutions

\section{Results and discussions}

\subsection{Parameter space}

The control parameters in this system are: the Reynolds number $\left(R e_{f}\right)$, the dimensionless plunging amplitude $(A / L)$, the pitching amplitude $\left(A_{\theta}\right)$, the mass ratio $(\beta)$, and the dimensionless bending rigidity $(\gamma)$. The parameter values used in the simulation are listed in Table 2.

Table 2 The physical parameters used in the simulations

\begin{tabular}{cccccc}
\hline$R e_{f}$ & $A / L$ & $A_{\theta}$ & $\Delta \phi$ & $\beta$ & $\gamma$ \\
\hline 300 & $\begin{array}{c}0.02-0 . \\
22\end{array}$ & $0^{\circ}-60^{\circ}$ & $0^{\circ}-360^{\circ}$ & 0.01 & $\begin{array}{c}10^{-3}-10 \\
3\end{array}$ \\
\hline
\end{tabular}

It is seen that very broad ranges of the dimensionless bending rigidity and the phase offset are considered in this study. The pitching and plunging amplitudes and the mass ratio lie in a reasonable range, as is consistent with those in some biological and mechanical systems that were studied previously. The Reynolds number selected in the simulation is of the order of $10^{2}$. This corresponds to the swimming of very small fish, say a fish with the 
length of $0.01 \mathrm{~m}$ cruising at the speed of one body length per second. There are two reasons for prescribing a relatively low Reynolds number in the simulation. First, the simulations at the Reynolds numbers comparable to those in the flapping-foil experiments or the swimming of large fish require a very high mesh resolution. This may render the broad parameter sweeps prohibitive. Second, in the range of moderate Reynolds numbers, the influence of the Reynolds number on the swimming performance is presumably to be much smaller than that of the kinematics. In view of the two reasons aforementioned, the Reynolds numbers considered in most of the computational studies fell within the range of $10^{2}-10^{3[5-11,19-21]}$.

\subsection{Quantities for characterizing performance, kine-} matics and wake structure

We use two dimensionless quantities, namely, the dimensionless cruising speed and the propulsive parameter to characterize the swimming performance. The dimensionless cruising speed is defined as

$$
U_{c}=\left[-\frac{1}{T_{f}} \int_{0}^{T_{f}}\left(\frac{\partial \boldsymbol{X}}{\partial t}\right)_{s=0} \mathrm{~d} t\right]
$$

where $T_{f}=1$ is the dimensionless flapping period. As an efficiency indicator for the self-propelled system, the propulsive parameter $\eta$ is defined as

$$
\eta=\frac{\beta}{2} \frac{U_{c}^{2}}{P_{s} T_{f}}
$$

where $P_{s}$ is the (dimensionless) input power that is required to produce the oscillation and the freeswimming ${ }^{[6]}$. Mathematically speaking, $P_{s}$ can be computed as

$$
P_{s}=\frac{1}{T_{f}} \int_{0}^{T_{f}}\left[\int_{0}^{1}\left(\boldsymbol{F} \cdot \frac{\partial \boldsymbol{X}}{\partial t}\right) \mathrm{d} s\right] \mathrm{d} t
$$

We use two parameters, namely, the dimensionless wave number and the slip ratio, to characterize the deformation pattern and the kinematics. The dimensionless wave number is defined as

$$
n=k L=\frac{2 \pi L}{\lambda}
$$

where $k$ is the wave number, $\lambda$ is the wave length. The slip ratio is defined as
$S L=\frac{\tilde{U}_{c}}{V}=\frac{U_{c} f L}{\lambda f}=\frac{n}{2 \pi} U_{c}$

where $\tilde{U}_{c}$ is the dimensional cruising velocity, $V$ is the speed of the wave propagation.

The Strouhal number, as an important parameter for characterizing the wake structure, is defined as

$S t=\frac{2 A_{t e} f}{\tilde{U}_{c}}=\frac{2 A_{t e}}{L} \frac{1}{U_{c}}$

where $A_{t e}$ is the plunging amplitude of the trailing edge. This parameter signifies the ratio of the lateral velocity at the trailing edge to the swimming velocity, or the ratio of the wake width to the streamwise spacing between two adjacent vortices.

\subsection{Influences of bending rigidity on performance}

To explore the effects of the bending rigidity on the swimming performance and the kinematics, all control parameters are fixed, except the dimensionless bending rigidity, which is allowed to vary in the range of $10^{-3}-10^{3}$. Two types of actuation, namely, the plunging-pitching actuation and the plunging-only actuation, are considered here. The fixed parameter values for the plunging-pitching cases are: $A / L=0.05, \quad A_{\theta}=20^{\circ}, \Delta \phi=90^{\circ}$. For the plunging-only cases, $A_{\theta}$ is set to zero and the rest parameters are the same as those for the plunging-pitching cases.

Figure 5(a) shows the dimensionless cruising speeds as a function of the dimensionless bending rigidity, for both types of actuation. In the curve for the plunging-only actuation, three distinct peaks are observed at $\gamma=0.005, \gamma=0.05$ and $\gamma=2.5$, respectively. These three peaks correspond to the third, second and first resonant modes, respectively. In the curve for the plunging-pitching actuation, the peaks corresponding to the second and third resonant modes are still observed. However, the peak corresponding to the first resonant mode does not appear and a broad plateau is observed instead when the dimensionless rigidity exceeds 10 . This marked difference between the performance characteristics of the two types of actuation is consistent with the finding reported by Lauder et al. ${ }^{[12]}$. Figure 5(b) shows the efficiency as a function of the dimensionless rigidity, for the two types of actuation. It is seen that for the plunging-only actuation, three efficiency peaks are observed at the rigidities corresponding to the peak cruising speeds. For the plunging-pitching actuation, besides the two efficiency peaks corresponding to the peak cruising 
speeds for the second and third resonant modes, another efficiency peak is observed at $\gamma=0.4$. By comparing the efficiencies achieved in the two types of actuation, it is found that the plunging-pitching actuation is superior to the plunging-only actuation across a wide range of rigidity. The only exception is found in the narrow rigidity range of $1<\gamma<10$. This range centers around the rigidity where the peak cruising speed for the first resonant mode in the plunging-only cases is achieved.
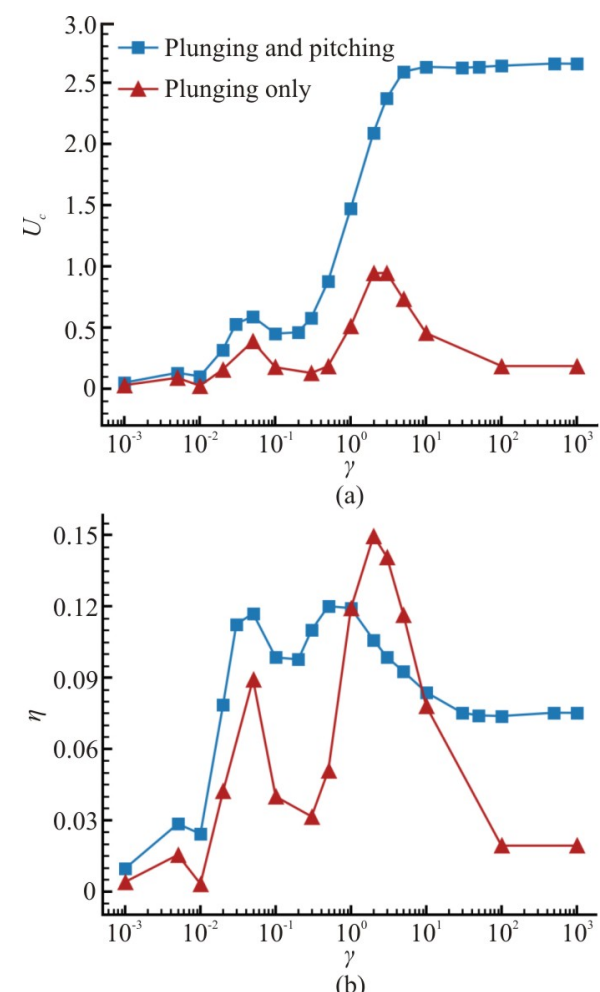

Fig. 5 (Color online) Variations of dimensionless cruising speed (a) and efficiency (b) against dimensionless bending rigidity

From the results shown in Fig. 5, the advantages of adding the pitching motion to the actuation can be summarized as follows. First, a much higher cruising speed can be achieved by imparting more energy to the filament and the fluid, with the extra benefit of efficiency enhancement (except within the narrow rigidity range of $1<\gamma<10)$. Second, the decrease of the cruising speed at a high rigidity can be avoided. It should also be noted that within the rigidity range of $1<\gamma<10$, the plunging-only actuation may be preferred if a relatively low cruising speed is tolerable and a high efficiency is the priority.

The deformation patterns (the amplitude envelopes) at some selected rigidities are shown in Fig. 6. It is seen that the deformation mode transits from the third mode to the second mode and then to the first mode, with increased bending rigidity. It is reasonable to believe that the transition of the deformation mode occurs when the actuation frequency approaches the natural frequencies of the specific order. At $\gamma=0.01$ and $\gamma=0.1$, the undulatory swimming style is observed. The deformation pattern produced by the plunging-only actuation is close to a standing-wave, while that produced by the plunging-pitching actuation is close to a traveling wave. Since a traveling wave is the key factor for the effective locomotion in the undulatory swimming ${ }^{[18]}$, the plunging-pitching actuation always produces a higher cruising speed. At $\gamma=1$, the oscillatory swimming style is observed. The higher cruising speed produced by the plunging-pitching actuation is mainly due to the larger flapping amplitude in the posterior part of the filament. At $\gamma=10$, the filament practically behaves like a rigid plate. For the plunging-only actuation, the decrease of the cruising speed at such a high rigidity (in comparison with the cruising speed achieved at $\gamma=1)$ is caused by the drastic reduction of the projected area in the vertical direction. Under such circumstances, the projected pressure force in the horizontal direction is close to zero. Thus, the pressure difference across the upper and lower surfaces cannot be effectively utilized to generate the propulsive force. In contrast, for the plunging-pitching actuation, the cruising speed keeps increasing with increased rigidity until a plateau region is reached, because both the trailing edge amplitude and the projected area in the vertical direction keep increasing with increased rigidity.

Here we also quantitatively compare the kinematics of the flexible filaments with those of animal swimmers. The variations of the dimensionless wave number and the slip ratio against the rigidity are shown in Fig. 7. As can be seen, for the plunging-only actuation, the dimensionless wave number and the slip ratio lie in the ranges of 0.01-9.09 and 0.0002-0.24, respectively. For the plunging-pitching actuation, the dimensionless wave number and the slip ratio lie in the ranges of $1.4-9.35$ and $0.08-0.86$, respectively. The data for the kinematics of animal swimmers were collected in several Refs. [28-31]. For anguilliform (eel-like) swimmers, the dimensionless wave number lies in the range of 6.28-20, while the slip ratio lies in the range of $0.5-0.75$. For carangiform (mackerel-like) swimmers, the dimensionless wave number is close to 6.28 , while the slip ratio is around 0.83 . Thus, the kinematics produced by the plunging-pitching actuation are closer to those of animal swimmers, as compared with those produced by the plunging-only actuation. If the plunging-pitching actuation is used to reproduce the kinematics similar to those of animal swimmers, the rigidity requirements stem from the 


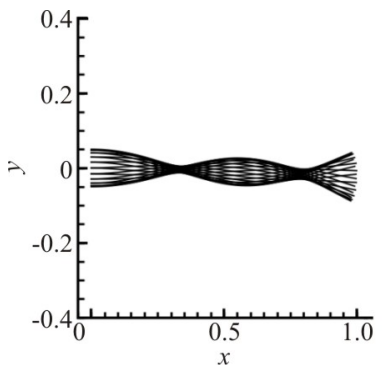

(a) $\gamma=0.01, A_{\theta}=0^{\circ}$

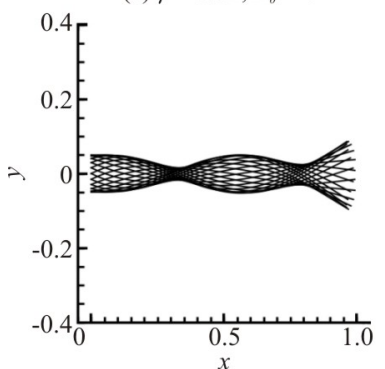

(e) $\gamma=0.01, A_{\theta}=20^{\circ}$

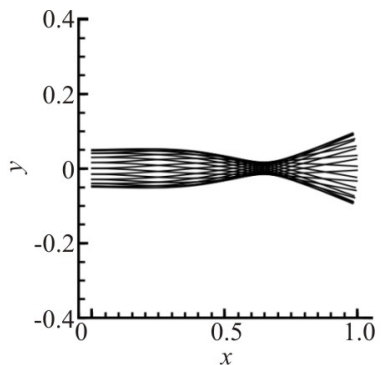

(b) $\gamma=0.1, A_{\theta}=0^{\circ}$

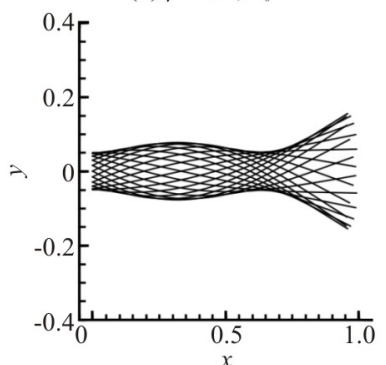

(f) $\gamma=0 . \stackrel{x}{1}, A_{\theta}=20^{\circ}$

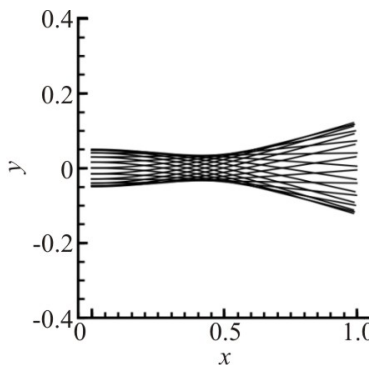

(c) $\gamma=\stackrel{x}{1}, A_{\theta}=0^{\circ}$

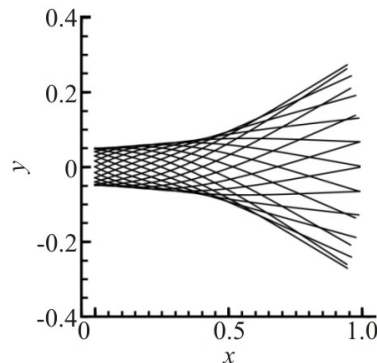

(g) $\gamma=1, A_{\theta}=20^{\circ}$

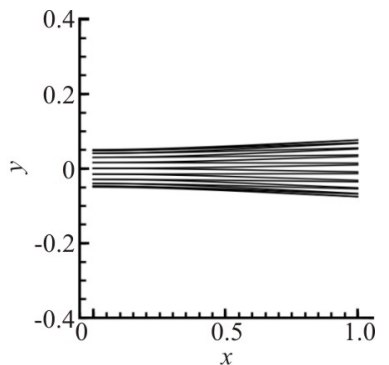

(d) $\gamma=10, A_{\theta}=0^{\circ}$

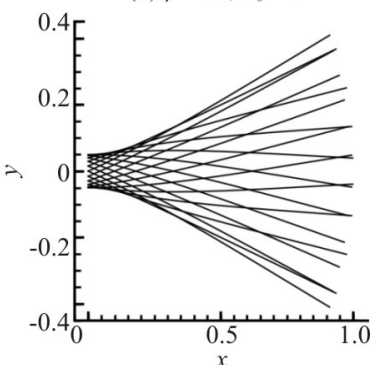

(h) $\gamma=10, A_{\theta}=20^{\circ}$

Fig. 6 Amplitude envelopes for the filaments with four different dimensionless bending rigidities. In each case, 20 filament shapes obtained at a time interval of 0.05 are superimposed on top of each other. (a)-(d): plunging-only actuation with $A / L=0.05$, (e)-(h): plunging-pitching actuation with $A / L=0.05, A_{\theta}=20^{\circ}, \Delta \phi=90^{\circ}$

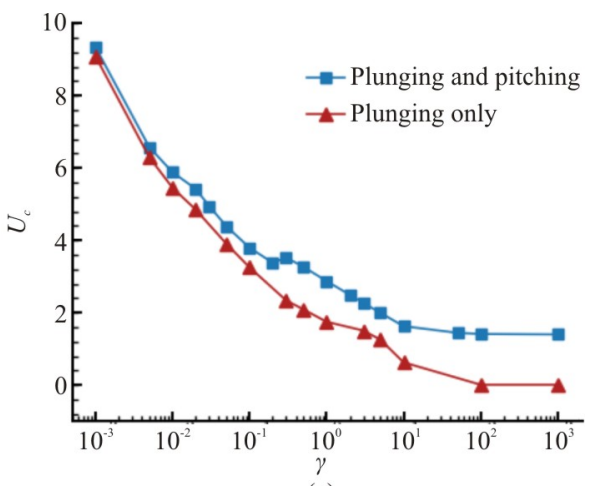

(a)

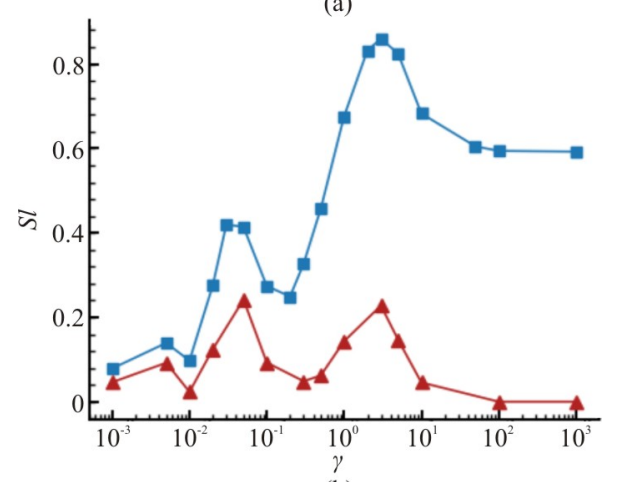

(b)

Fig. 7 (Color online) Variations of dimensionless wave number (a) and slip ratio (b) against dimensionless bending rigidity

similarities of the dimensionless wave number and the slip ratio are $\gamma \leq 0.03$ and $0.4 \leq \gamma \leq 2$, respectively (cf. Figs. 7(a), 7(b)). The above conflicting requirements indicate that the kinematics of swimming animals cannot be reproduced by a flexible filament with any given rigidity.

The unsuccessful results of emulating the kinematics of animal swimmers by the flexible filament can be attributed to several fundamental differences between the two systems. First, for the filament model the actuation is localized, while for animal swimmers the muscles are distributed along the body. Second, for the filament model the actuation motions are of sinusoidal form, while the actuation motions of animal swimmers are more complex (not necessarily in sinusoidal form). Third, for the filament model the material properties (such as the rigidity and the mass) are assumed to be homogeneous, while for animal swimmers the material properties are nonuniformly distributed. However, the results of previous studies indicated that the carangiform swimming style can be accurately emulated (even with a localized and sinusoidal actuation), if homogeneous rigidity and mass were permitted ${ }^{[19]}$. It is not surprising if the anguilliform swimming style can also be successfully reproduced by tuning the inhomogeneous material properties.

Figure 8 shows the variation of the Strouhal number $(S t)$ against the dimensionless rigidity. For both types of actuation, a similar trend is observed in the variation of $S t$ against the increasing rigidity. At a low rigidity, $S t$ becomes relatively high due to the low cruising speed. With increasing rigidity, $S t$ begins to decrease and finally reaches a plateau region 
when the rigidity is sufficiently high. However, some differences between the $S t$ curves for the two types of actuation can still be seen. For the plungingpitching actuation, the $S t$ curve is very smooth and only minor fluctuations are observed. For the plunging-only actuation, the fluctuations are much larger and a distinct local peak is seen near $\gamma=0.3$. The peak $S t$ at this rigidity results from the local minimum of the cruising speed (cf. Fig. 5(a)). The asymptotic $S t$ value at an extremely high rigidity for the plunging-pitching actuation is around 0.5 , which is much higher than the value for the plunging-pitching actuation (which is around 0.25 ).

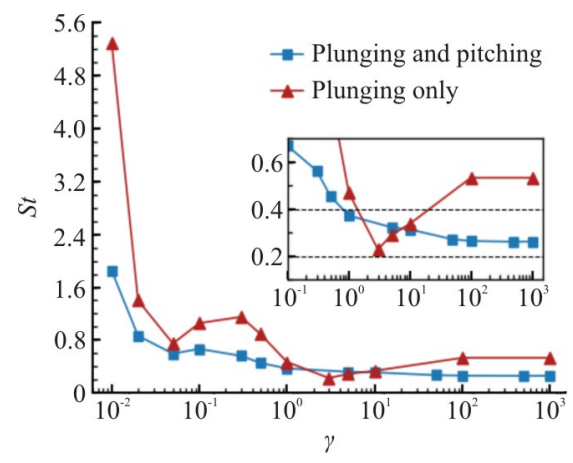

Fig. 8 (Color online) Variation of Strouhal number against dimensionless bending rigidity

We also compare the St for the plungingpitching actuation with those of some animal swimmers (and flyers). Some evidence is found that St in the cruising swimming and flight of animals converges on a narrow range of $0.2-0.4^{[32]}$. This range is supposed to be linked with the peak propulsive efficiency in rigid foils in the pitching and plunging motions ${ }^{[33]}$. However, some recent studies indicate that only non-anguilliform swimmers indeed cruise at $S t \approx 0.3$, while anguilliform swimmers tend to have much higher $S t$ values ${ }^{[34-37]}$. In this study, we find that for the deformation patterns of second or higher bending mode $(\gamma \leq 0.01), S t$ is well above the range of 0.2-0.4. This is consistent with the results of a numerical study on the undulatory swimming at low (cruising) Reynolds numbers ${ }^{[38]}$. For semi-rigid and rigid filaments $(\gamma \geq 1), S t$ falls in the narrow range of 0.2-0.4 and varies little with the rigidity. By comparing Fig. 8 with Fig. 5(b), we can see that the results of the present study challenge the suggestion that the St range of 0.2-0.4 is associated with the high efficiency. In other words, our results suggest that the narrowly converged range of $S t$ in animal swimmers (and flyers) may just be the natural outcome of less deformed bodies in self-propelled swimming (rather than the result of painstaking tuning).

\subsection{Influences of actuation parameters on swimming performance}

In this section, we explore the influences of actuation parameters, including the phase offset $\Delta \phi$, the dimensionless plunging amplitude $A / L$ and the pitching amplitude $A_{\theta}$, on the swimming performance. Three dimensionless rigidities, namely, 0.005 , 0.1 and 1 , are selected in the investigation. These three rigidities correspond to the deformation patterns of the third, second and first binding mode, respectively. For each rigidity, parameter sweeps are performed in the 2-D spaces of $(A / L, \Delta \phi)$ and $\left(A_{\theta}, \Delta \phi\right)$. In the space of $(A / L, \Delta \phi), A_{\theta}$ is fixed to $20^{\circ}$, while in the space of $\left(A_{\theta}, \Delta \phi\right), A / L$ is fixed to 0.05 . In all cases considered in the investigation, the Reynolds number $\operatorname{Re}_{f}$ and the mass ratio $\beta$ are fixed to 300 and 0.01 , respectively.

The contours of the dimensionless cruising speed and the efficiency in the parameter spaces of $(A / L, \Delta \phi)$ and $\left(A_{\theta}, \Delta \phi\right)$ are shown in Figs. 9-11, for the three selected rigidities, respectively. The influences of the actuation parameters on the cruising speed can be summarized as follows. For the given plunging and pitching amplitudes, the maximum and minimum cruising speeds are reached approximately at $\Delta \phi=0^{\circ}$ (or $\Delta \phi=360^{\circ}$ ) and $\Delta \phi=180^{\circ}$, respectively. For the given phase offset, the cruising speed monotonically increases with the increased pitching or plunging amplitude. At certain plunging and pitching amplitudes, a negative cruising speed can be produced if the phase offset is close to $180^{\circ}$. A negative cruising speed signifies the backward swimming (i.e., swimming towards the right), which is usually accompanied with a relatively small velocity. This abnormal swimming mode was also reported in the study of self-propelled rigid foils driven by plunging-pitching motions ${ }^{[39]}$.

The influences of the actuation parameters on the efficiency are more complex. Generally speaking, the in-phase and anti-phase actuations correspond to the highest and the lowest efficiencies, respectively. Therefore, the aforementioned abnormal swimming mode is usually accompanied with a low efficiency. However, some exceptions are also observed in the second and first bending modes. For example, the highest efficiency can be achieved at $\Delta \phi=45^{\circ}$ (cf. Figs. 10(b), 11(b)). As to the influences of the flapping amplitudes on the efficiency, the increase of the efficiency with the increased plunging or pitching amplitude is only found in the third bending mode (cf. Figs. 9(b), 9(d)). In the other two bending modes, the 


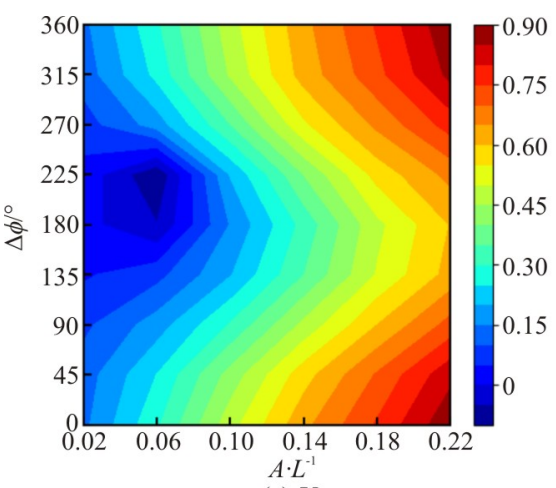

(a) $U_{c}$
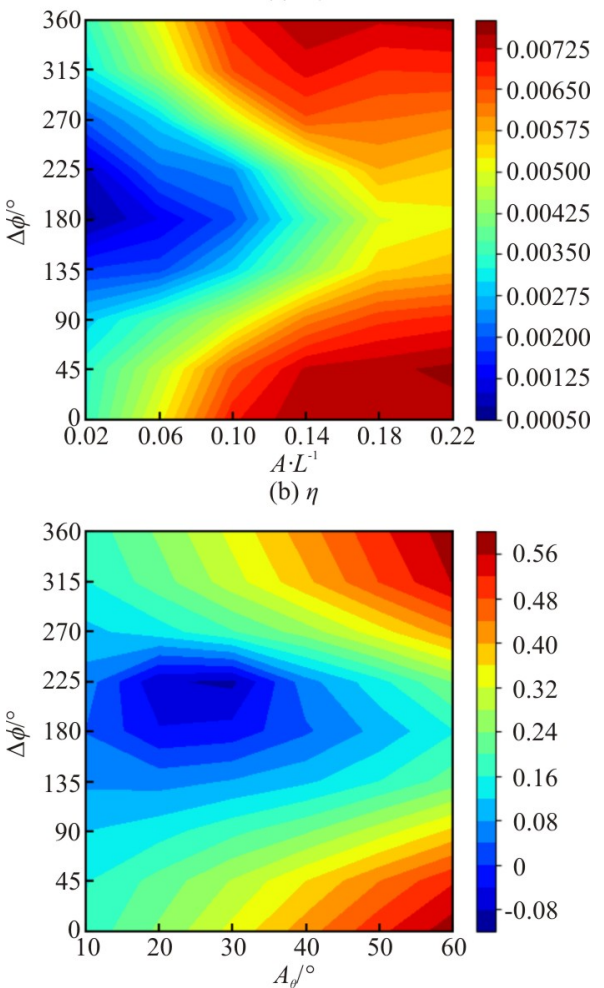

(c) $U_{c}$

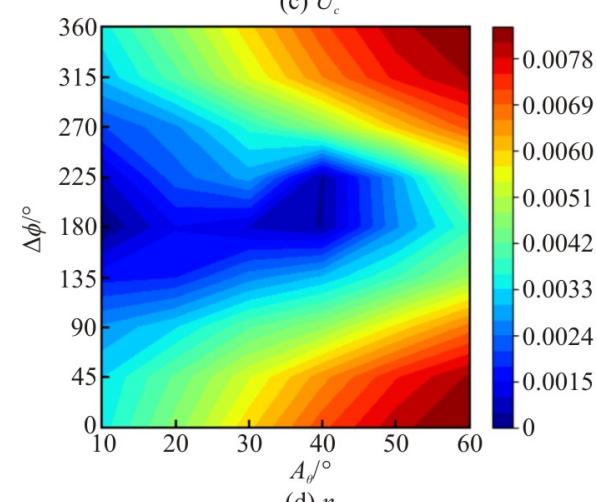

(d) $\eta$

Fig. 9 (Color online) Contours of dimensionless cruising speed and efficiency in the parameter spaces of $(A / L, \Delta \phi)$ and $\left(A_{\theta}, \Delta \phi\right)$, for $\gamma=0.005$

highest efficiency can be achieved at a moderate or low flapping amplitude (cf. Figs. 10(d), 11(d)). By

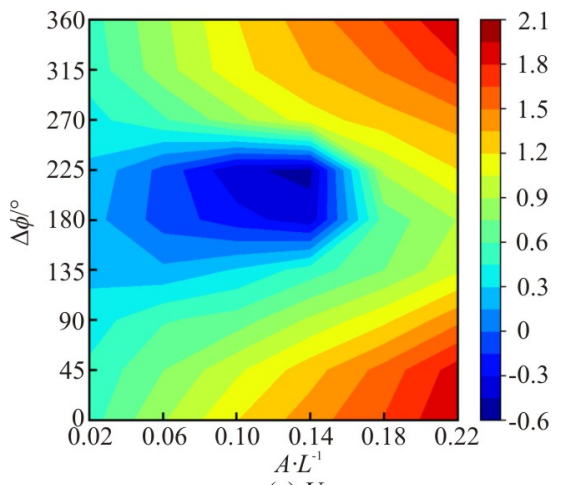

(a) $U_{c}$

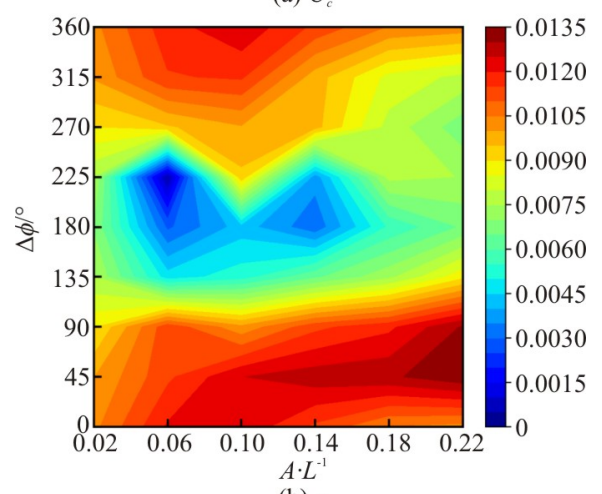

(b) $\eta$

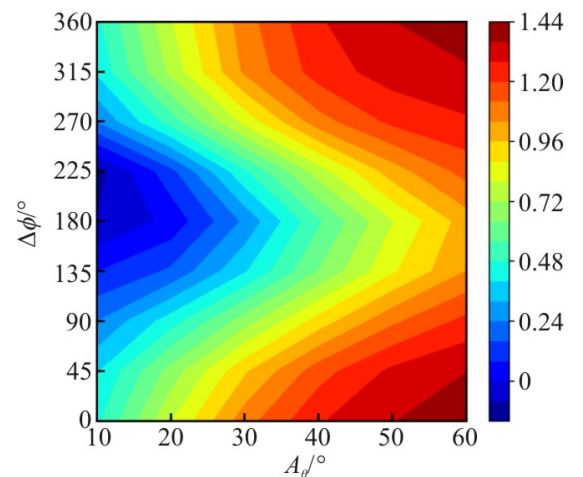

(c) $U_{c}$

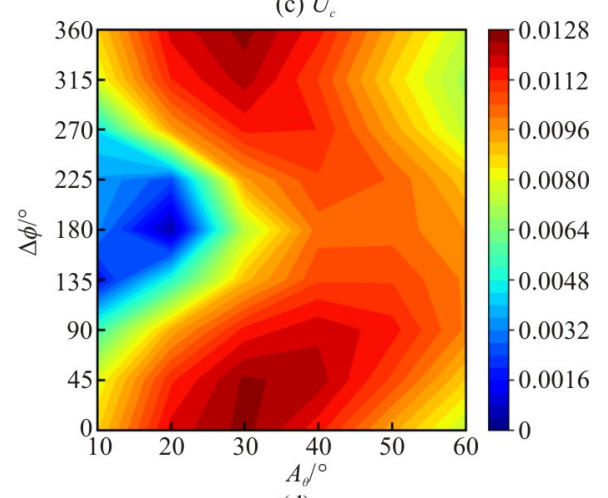

(d) $\eta$

Fig. 10 (Color online) Contours of dimensionless cruising speed and efficiency in the parameter spaces of $(A / L, \Delta \phi)$ and $\left(A_{\theta}, \Delta \phi\right)$, for $\gamma=0.1$

comparing the efficiency contours in the space of $\left(A_{\theta}, \Delta \phi\right)$ for the three selected rigidities, it is also 


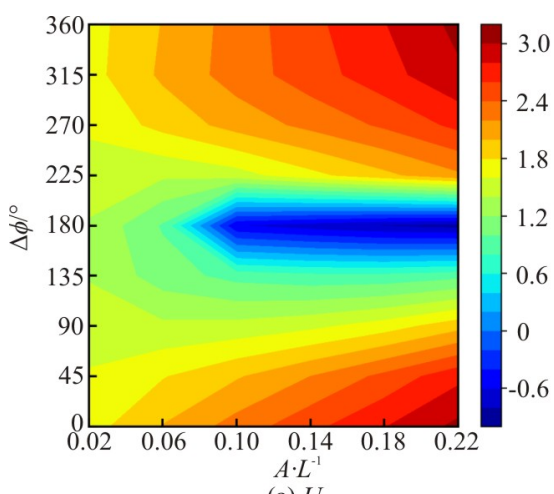

(a) $U_{c}$
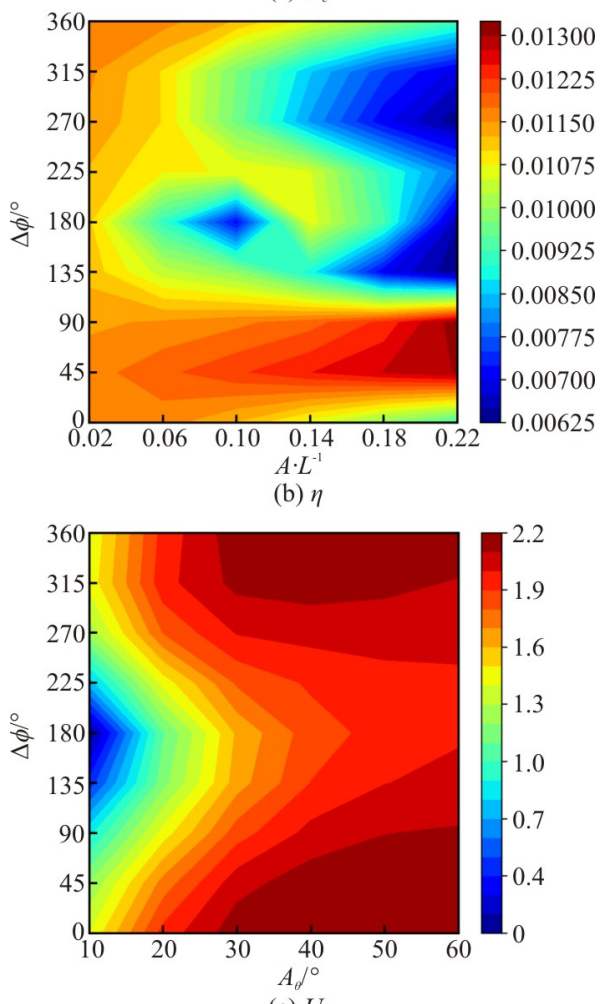

(c) $U_{c}$

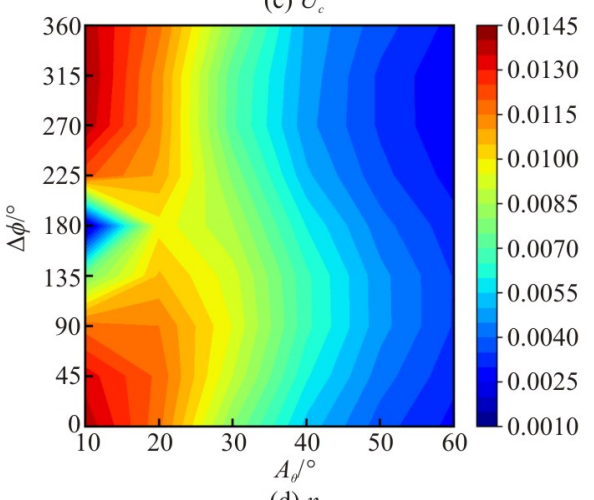

(d) $\eta$

Fig. 11 (Color online) Contours of dimensionless cruising speed and efficiency in the parameter spaces of $(A / L, \Delta \phi)$ and $\left(A_{\theta}, \Delta \phi\right)$, for $\gamma=1.0$

found that the optimal pitching amplitude (for achieving the highest efficiency) decreases with in- crease rigidity.

Here an explanation for the great contrast between the performances of the in-phase and anti-phase actuations is provided. In Fig. 12, the deformation patterns (the amplitude envelopes) under the two types of actuation are compared and two major differences are found. First, it is seen that for all three selected rigidities, the in-phase actuation tends to produce a much larger flapping amplitude along the entire body, as compared with the anti-phase actuation. Second, the deformation form produced by the in-phase actuation is close to a traveling wave, while that produced by the anti-phase actuation is close to a standing wave. As is pointed out in Section 3.3, the high flapping amplitude and the formation of a traveling wave are the two key factors that can lead to a high cruising speed.

A comparison of the present results with the findings in other references is also presented here. The peak speeds for the self-propelled flexible filaments are always achieved at $\Delta \phi=0^{\circ}$ in the present study. This is consistent with the finding by Piñeirua et al. ${ }^{[22]}$ In Kim et al. ${ }^{[21]}$, the maximum speed was found to be achieved at $\Delta \phi=90^{\circ}$ (with given rigidity and flapping amplitudes). For the self-propelled rigid foils, the result of Lin et al. ${ }^{[39]}$ indicated that the phase offset of $\Delta \phi=60^{\circ}$ produced the maximum speed (with given flapping amplitudes). As for the efficiency, the present result indicates that the highest efficiency is obtained in the phase offset range of $0^{\circ}-90^{\circ}$, and the optimal phase offset depends on the values of the flapping amplitudes and the rigidity. In Piñeirua et al. ${ }^{[22]}$, Kim et al. ${ }^{[21]}$, the in-phase $\left(\Delta \phi=0^{\circ}\right)$ and the anti-phase $\left(\Delta \phi=180^{\circ}\right)$ actuations were found to be optimal for flexible filaments (with given rigidity and flapping amplitudes), respectively. For rigid foils, the result by Lin et al. ${ }^{[39]}$ indicated that the optimal phase offset (with given flapping amplitudes) was around $90^{\circ}$. Thus, it seems that the present finding is a reconciliation of the results reported by Piñeirua et al. ${ }^{[22]}$ and Lin et al. ${ }^{[39]}$, but is in a contrast to the result by Kim et al. ${ }^{[21]}$.

It should be noted that all results listed above are based on the self-propelled model, in which the propulsive parameter $\eta$ is used to quantify the efficiency. Some other studies were based on the "tethered" model placed in a flow with a prescribed speed and the Froude efficiency was used as the efficiency indicator. The results for the optimal phase offset (for achieving the highest efficiency) based such model are also listed here. Quinn et al. ${ }^{[40]}$ found that for the flexible filaments the optimal phase offset was in the range of $76^{\circ}-96^{\circ}$. For the rigid foils, it was shown many times that the optimal efficiency was 

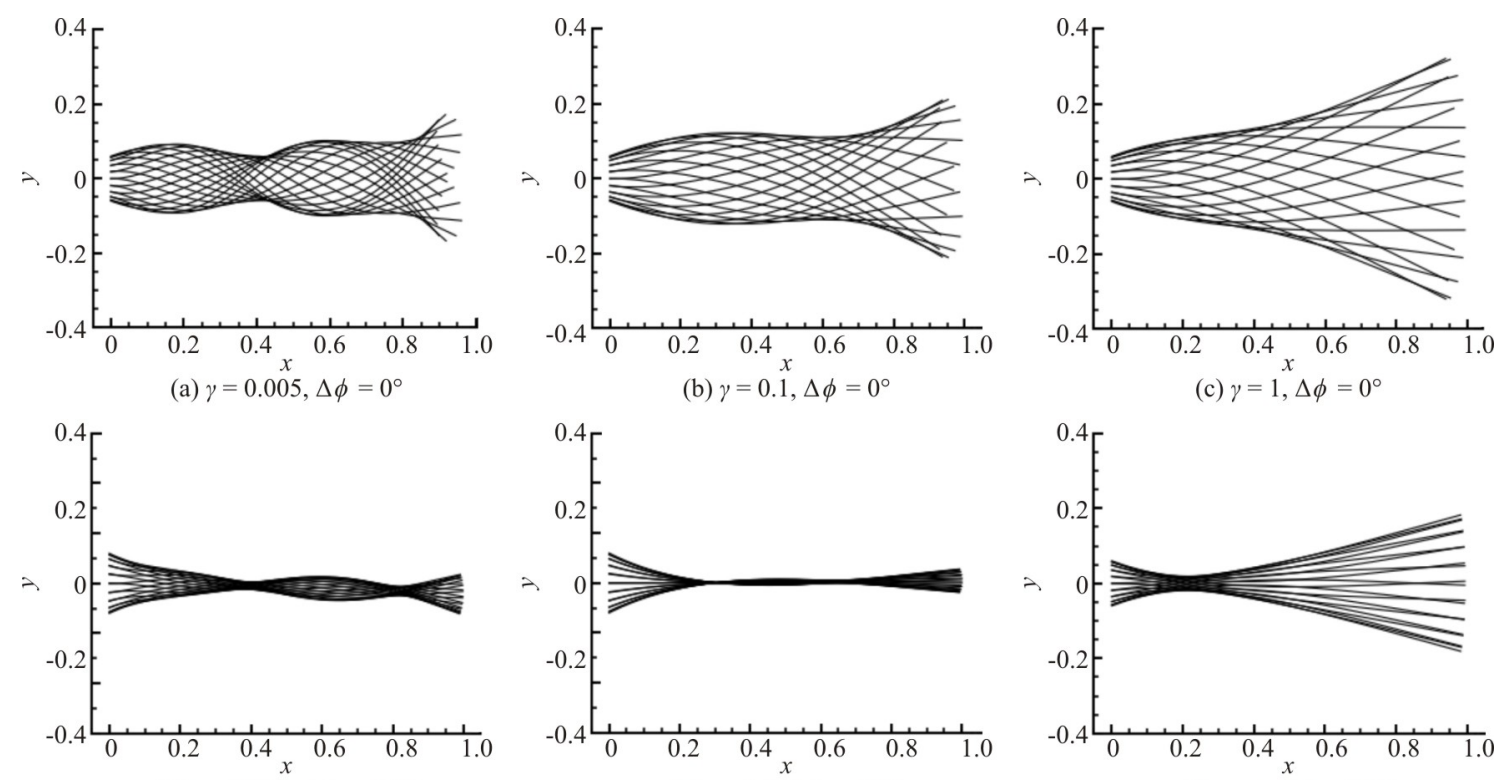

(d) $\gamma=0.005, \Delta \phi=180^{\circ}$

(e) $\gamma=0.1, \Delta \phi=180^{\circ}$

(f) $\gamma=1, \Delta \phi=180^{\circ}$

Fig. 12 Amplitude envelopes of the filaments produced by in-phase and anti-phase actuations, at three selected bending rigidities. In each case, 20 filament shapes obtained at a time interval of 0.05 are superimposed on top of each other. (a)-(c): in-phase actuation; (d)-(f): anti-phase actuation

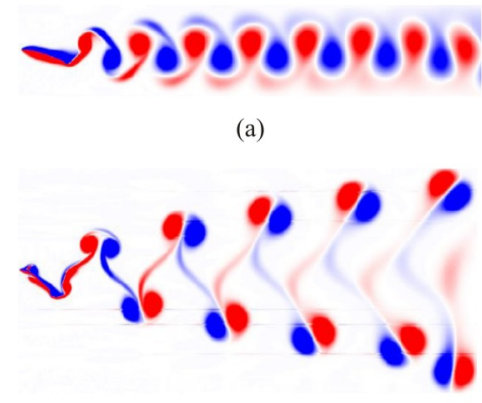

(c)

\section{ececerecerectegers}

(b)

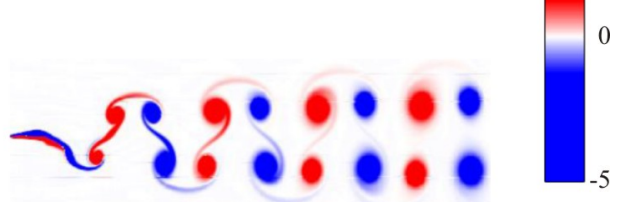

(d)

Fig. 13 (Color online) Wake structures of the swimming filament. (a) "2S" type (forward swimming mode): $\gamma=1, A / L=0.05, \quad A_{\theta}=15^{\circ}, \Delta \phi=90^{\circ},(\mathrm{b})$ "2S" type (backward swimming mode): $\gamma=1, A / L=0.18$, $A_{\theta}=20^{\circ}, \Delta \phi=180^{\circ}$, (c) "2P" type (two oblique lines of dipoles): $\gamma=1, A / L=0.05, A_{\theta}=50^{\circ}, \Delta \phi=90^{\circ}$, (d) "2P" type (two horizontal vortex streets): $\gamma=100, A / L=0.05, A_{\theta}=25^{\circ}, \Delta \phi=90^{\circ}$. The contours of dimensionless vorticity (vorticity scaled by $f$ ) are used to visualize the vortex structures

achieved at $\Delta \phi=270^{\circ}[41-43]$. In short, the inconsistencies regarding how the swimming performance is affected by the phase offset are still not fully resolved and further investigations are needed.

\subsection{Wake structures}

The observed wake structures can be classified into two categories, namely, " $2 \mathrm{~S}$ " and " $2 \mathrm{P}$ ", based on the number of vortices that are shed in one flapping period. Here the term " $2 S$ " refers to the situation in which two vortices of opposite sign are shed per flapping period, while the term " $2 \mathrm{P}$ " refers to the situation in which two pair of vortices are shed per flapping period. The wake structures of both types were widely reported in the previous studies on the rigid $^{[44]}$ and flexible flapping foils ${ }^{[6]}$.

Figures 13(a), 13(b) show two wake structures of the " $2 S$ " type. The only difference between them lies in the swimming direction. In Fig. 13(a), the foil moves towards the left when the actuation is applied at the leading edge (the left endpoint). This is the forward swimming mode which appears if the phase offset $\Delta \phi$ is kept away from $180^{\circ}$. In Fig. 13(b), the foil swims towards the right when the actuation is applied at the leading edge. This is the backward swimming mode, which only appears when the phase 
offset $\Delta \phi$ lies in a narrow range very close to $180^{\circ}$.

Figures 13(c), 13(d) show two wake structures of the "2P" type. The difference between them lies in the spatial organization of the vortices. In Fig. 13(c), the vortices are clustered along two oblique lines. Within each line, two adjacent vortices with opposite sign pair with each other to form dipoles. In Fig. 13(d), the vortices are clustered along two horizontally oriented parallel lines. Within each line, the vortices with opposite sign are positioned alternately in an equidistant fashion.

For the flapping-foil systems, asymmetric wakes (with respect to the centerline) were also widely reported in the literature ${ }^{[5-6,8,11,44-45]}$. In some studies, the material properties and the flapping amplitudes were found to have great influences on the wake symmetry property ${ }^{[11,45]}$. In the present work, only symmetric wakes have been observed. It seems that the selected parameter values in this work just happen to lie in the range where the wake symmetry breaking is suppressed. From Fig. 13, some distortions of the vorticity fields are found at the interfaces between coarse and fine meshes. The cause of such distortions has been explained before in Section 2.

\section{Conclusions}

Numerical simulation is conducted to study the self-propelled swimming of a flexible filament driven at the leading edge by coupled pitching and plunging motions. We focus on the influences of the rigidity and the actuation parameters on the swimming performance.

We examine the cruising speed and the efficiency for both the plunging-pitching and the plunging-only actuations across a wide range of rigidity. It is found that at an extremely low rigidity, no forward motion can be produced. The undulatory swimming mode is observed with the increase of rigidity. The swimming style gradually transits to the oscillatory mode if the rigidity is increased further. The filament behaves like a rigid flapping foil at an extremely high rigidity. Except for a very narrow range of rigidity, the plunging-pitching actuation enjoys a higher efficiency than the plunging-only actuation. The plungingpitching actuation can also prevent the decrease of the cruising speed at a high rigidity (known as one shortcoming of the plunging-only actuation).

The phase offset between the pitching and plunging motions and the flapping amplitudes are found to have great influences on the swimming performance. Generally speaking, the in-phase actuation signifies a high speed and a high efficiency, whereas the anti-phase actuation signifies a low speed and a low efficiency. The cruising speed monotonically increases with the increase of plunging or pitching amplitude. The relation between the efficiency and the flapping amplitudes is found to be very complex. The optimal flapping amplitudes for achieving the highest efficiency strongly depend on the rigidity of the filament.

Two types of wake structure, namely, " $2 \mathrm{~S}$ " and " $2 \mathrm{P}$ ", are observed. Within the parameter range of the present study, the wake symmetry breaking is not detected.

Based on the experiences gained on the limited cases that are simulated here, the ideal situations for the efficiency optimization are summarized as follows. First, moderate flexibilities are preferred. At moderate flexibilities, the deformation modes of the first or second order are produced and the flapping amplitudes of the tail are much higher than those of the head. Second, the ideal actuation motion should have a phase difference close to zero, the highest possible plunging amplitude (within the range considered here) and a pitching amplitude which is not too high (less than $40^{\circ}$ ). The best pitching amplitude strongly depends on the flexibility and decreases with decreasing flexibility.

\section{Acknowledgements}

This work was supported by the Chinese Academy of Sciences (Grant Nos. XDB22040104, XDA22040203). We would like to thank Dr. Long-zhen Dai for the development of the FSI code, and the National Supercomputing Center in Tianjin (NSCC-TJ) for the allocation of computing time.

\section{References}

[1] Lauder G. V., Anderson E. J., Tangorra J. et al. Fish biorobotics: Kinematics and hydrodynamics of selfpropulsion [J]. Journal of Experimental Biology, 2007, 210(16): 2767-2780.

[2] Wang S. Z., He G. W., Zhang X. Self-propulsion of flapping bodies in viscous fluids: Recent advances and perspectives [J]. Acta Mechanica Sinica, 2016, 32(6): 980-990.

[3] Liu C., Hu C. An efficient immersed boundary treatment for complex moving object [J]. Journal of Computational Physics, 2014, 274: 654-680.

[4] Gronskis A., Artana G. A simple and efficient direct forcing immersed boundary method combined with a high order compact scheme for simulating flows with moving rigid boundaries [J]. Computers and Fluids, 2016, 124: 86-104.

[5] Hua R. N., Zhu L., Lu X. Y. Locomotion of a flapping flexible plate [J]. Physics of Fluids, 2013, 25(12): 121901.

[6] Zhu X., He G., Zhang X. Numerical study on hydrodynamic effect of flexibility in a self-propelled plunging foil [J]. Computers and Fluids, 2014, 97: 1-20.

[7] Olivier M., Dumas G. Effects of mass and chordwise flexibility on 2D self-propelled flapping wings [J]. Journal of Fluids and Structures, 2016, 64: 46-66. 
[8] Zhang D., Pan G., Chao L. et al. Effects of Reynolds number and thickness on an undulatory self-propelled foil [J]. Physics of Fluids, 2018, 30(7): 071902.

[9] Hoover A. P., Cortez R., Tytell E. D. et al. Swimming performance, resonance and shape evolution in heaving flexible panels [J]. Journal of Fluid Mechanics, 2018, 847: 386-416.

[10] Ryu J., Park S. G., Huang W. X. et al. Hydrodynamics of a three-dimensional self-propelled flexible plate [J]. Physics of Fluids, 2019, 31(2): 021902.

[11] Zhu X., He G., Zhang X. How flexibility affects the wake symmetry properties of a self-propelled plunging foil [J]. Journal of Fluid Mechanics, 2014, 751: 164-183.

[12] Lauder G. V., Madden P. G. A., Tangorra J. L. Bioinspiration from fish for smart material design and function [J]. Smart Materials and Structures, 2011, 20(9): 094014.

[13] Lauder G. V., Lim J., Shelton R. et al. Robotic models for studying undulatory locomotion in fishes $[\mathrm{J}]$. Marine Technology Society Journal, 2011, 45(4): 41-55.

[14] Lauder G. V., Flammang B., Alben S. Passive robotic models of propulsion by the bodies and caudal fins of fish [J]. Integrative and Comparative Biology, 2012, 52(5): 576-587.

[15] Shelton R. M., Thornycroft P. J. M., Lauder G. V. Undulatory locomotion of flexible foils as biomimetic models for understanding fish propulsion [J]. Journal of Experimental Biology, 2014, 217(12): 2110-2120.

[16] Feilich K. L., Lauder G. V. Passive mechanical models of fish caudal fins: Effects of shape and stiffness on self-propulsion [J]. Bioinspiration and Biomimetics, 2015, 10(3): 036002.

[17] Lucas K. N., Thornycroft P. J. M., Gemmell B. J. et al. Effects of non-uniform stiffness on the swimming performance of a passively-flexing, fish-like foil model [J]. Bioinspiration and Biomimetics, 2015, 10(5): 056019.

[18] Ramananarivo S., Godoy-Diana R., Thiria B. Passive elastic mechanism to mimic fish-muscle action in anguilliform swimming [J]. Journal of the Royal Society Interface, 2013, 10 (88): 20130667.

[19] Dai L., He G., Zhang X. et al. Stable formations of selfpropelled fish-like swimmers induced by hydrodynamic interactions [J]. Journal of Royal Society Interface, 2018, 15(147): 20180490

[20] Dai L., He G., Zhang X. et al. Intermittent loco-motion of a fish-like swimmer driven by passive elastic mechanism [J]. Bioinspiration and Biomimetics, 2018, 13(5): 056011.

[21] Kim B., Park S. G., Huang W. et al. Self-propelled heaving and pitching flexible fin in a quiescent flow [J]. International Journal of Heat and Fluid Flow, 2016, 62: 273-281.

[22] Piñeirua M., Thiria B., Godoy-Diana R. Modelling of an actuated elastic swimmer [J]. Journal of Fluid Mechanics, 2017, 829: 731-750.

[23] Huang W. X., Shin S. J., Sung H. J. Simulation of flexible filaments in a uniform flow by the immersed boundary method [J]. Journal of Computational Physics, 2007, 226(2): 2206-2228.

[24] Wang S. Z., Zhang X. An immersed boundary method based on discrete stream function formulation for two- and three-dimensional incompressible flows [J]. Journal of Computational Physics, 2011, 230(9): 3479-3499.

[25] Wang S., He G., Zhang X. Parallel computing strategy for a flow solver based on immersed boundary method and discrete stream-function formulation [J]. Computers and Fluids, 2013, 88: 210-224.

[26] Dai L., He G., Zhang X. Self-propelled swimming of a flexible plunging foil near a solid wall [J]. Bioinsipration and Biomimetics, 2016, 11(4): 046005.

[27] Yang X., Zhang X., Li Z. et al. A smoothing technique for discrete delta functions with application to immersed boundary method in moving boundary simulations [J]. Journal of Computational Physics, 228(20): 7821-7836.

[28] Kern S., Koumoutsakos P. Simulations of optimized anguilliform swimming [J]. Journal of Experimental Biology, 2006, 209(24): 4841-4857.

[29] Videler J. J. Fish swimming [M]. Berlin, Germany: Springer, 1993

[30] Piñeirua M., Thiria B., Godoy-Diana R. Resistive thrust production can be as crucial as added mass mechanisms for inertial undulatory swimmers [J]. Physical Review E, 2015, 92(2): 021001.

[31] Cui Z., Yang Z. X., Shen L. et al. Complex modal analysis of the movements of swimming fish propelled by body and/or caudal fin [J]. Wave Motion, 2018, 78: 83-97.

[32] Taylor G. K., Nudds R. L., Thomas A. L. R. Flying and swimming animals cruise at a Strouhal number tuned for high power efficiency [J]. Nature, 2003, 425(6959): 707-711.

[33] Triantafyllou M., Triantafyllou G., Gopalkrishnan R. Wake mechanics for thrust generation in oscillating foils [J]. Physics of Fluids A: Fluid Dynamics, 1991, 3(12): 2835-2837.

[34] Borazjani I., Sotiropoulos F. Numerical investigation of the hydrodynamics of carangiform swimming in the transitional and inertial flow regimes [J]. Journal of Experimental Biology, 2008, 211(10): 1541-1558.

[35] Borazjani I., Sotiropoulos F. Numerical investigation of the hydrodynamics of anguilliform swimming in the transitional and inertial flow regimes [J]. Journal of Experimental Biology, 2009, 212(4): 576-592.

[36] Nangia N., Bale R., Chen N. et al. Optimal specific wavelength for maximum thrust production in undulatory propulsion [J]. PLoS One, 2017, 12(6): e0179727.

[37] Reid D. A. P., Hildenbrandt H., Padding J. T. et al. Fluid dynamics of moving fish in a two-dimensional multiparticle collision dynamics model [J]. Physical Review E, 2012, 85(2): 021901

[38] Weerden J. F., Reid D. A. P., Hemelrijk C. K. A metaanalysis of steady undulatory swimming [J]. Fish and Fisheries, 2014, 15(3): 397-409.

[39] Lin X., Wu J., Zhang T. Performance investigation of a self-propelled foil with combined oscillating motion in stationary fluid [J]. Ocean Engineering, 2019, 175: 33-49.

[40] Quinn D. B., Lauder G. V., Smits A. J. Maximizing the efficiency of a flexible propulsor using experimental optimization [J]. Journal of Fluid Mechanics, 2015, 767: 430- 448.

[41] Isogai K., Shinmoto Y., Watanabe Y. Effects of dynamic stall on propulsive efficiency and thrust of flapping airfoil [J]. AIAA Journal, 1993, 37(10): 1145-1151.

[42] Ramamurti R., Sandberg W. Simulation of flow about flapping airfoils using finite element incompressible flow solver [J]. AIAA Journal, 2001, 39(2): 253-260.

[43] Guglielmini L., Blondeaux P. Propulsive efficiency of oscillating foils [J]. European Journal of Mechanics, B/Fluids, 2004, 23(2): 255-278.

[44] Schnipper T., Andersen A., Bohr T. Vortex wakes of a flapping foil [J]. Journal of Fluid Mechanics, 2009, 633: 411-423.

[45] Marais C., Thiria B., Wesfreid J. E. et al. Stabilizing effect of flexibility in the wake of a flapping foil [J]. Journal of Fluid Mechanics, 2012, 710: 659-669. 